\title{
[Poster] Association between prenatal exposure to ambient particulate matter (PM2.5) and adverse birth outcomes in Nepal and Bangladesh using the prediction model for daily PM2.5 concentrations matched with demographic and health survey data: spatio-temporal analysis
} \author{
multisource satellite data including Sentinel-5P \\ Ayako Ogawa Kawano \\ School of Public Health, University of California, Berkeley \\ ayako_kawano@berkeley.edu
}

Predicting PM2.5 in Nepal and Bangladesh using XGBoost, LightGBM, and deep learning with

\begin{abstract}
There is growing evidence on associations of prenatal exposure to ambient particulate matter (PM2.5) with adverse birth outcomes including preterm birth and low birth weight; however, no previous studies have examined such associations in Nepal and Bangladesh due to the lack of resources that affect PM2.5 exposure data collection. This study aims at conducting a spatial-temporal analysis of an association between prenatal exposure to PM2.5 and preterm birth and term low birth weight in Nepal and Bangladesh by using the prediction model for PM2.5 daily concentrations that is geographically matched with Demographic and Health Survey (DHS) data. The main purpose of this poster is to introduce the overall picture of the study and present the materials, methods, and results of the first component of the study: (1) Develop a prediction model for daily PM2.5 concentrations in Nepal and Bangladesh. Contrary to traditional predictive models using satellite-detected Aerosol Optical Depth (AOD) for PM2.5 predictions, our model used the satellite-detected air pollution data (Sentinel-5P). The model performed well overall $\left(\mathrm{R}^{2}=0.79\right)$, for a post-monsoon period $\left(\mathrm{R}^{2}=\right.$ $0.79)$, and winter $\left(R^{2}=0.74\right)$, but worked poorly for summer $\left(R^{2}=\right.$ $0.61)$ and a monsoon period $\left(R^{2}=0.46\right)$. The model can be further improved by adding input features such as distance to sources of air pollution, local traffic-related data, and ground-level air pollutants that can be statistically computed from relevant column values obtained from Sentinel-5P.
\end{abstract}

\section{CCS CONCEPTS}

- Machine learning; • Modeling and simulation; • Estimation;

This work is licensed under a Creative Commons Attribution International 4.0 License.

COMPASS '21, June 28-7uly 02, 2021, Virtual Event, Australia

(C) 2021 Copyright held by the owner/author(s).

ACM ISBN 978-1-4503-8453-7/21/06.

https://doi.org/10.1145/3460112.3472312

\section{KEYWORDS}

Predictive modeling, Supervised machine learning, Deep learning, Global health, Ambient air pollution, Fine particulate matter, Preterm birth, Low birth weight, Developmental Origins of Health and Disease

\section{ACM Reference Format:}

Ayako Ogawa Kawano. 2021. [Poster] Association between prenatal exposure to ambient particulate matter (PM2.5) and adverse birth outcomes in Nepal and Bangladesh using the prediction model for daily PM2.5 concentrations matched with demographic and health survey data: spatio-temporal analysis: Predicting PM2.5 in Nepal and Bangladesh using XGBoost, LightGBM, and deep learning with multisource satellite data including Sentinel5P. In ACM SIGCAS Conference on Computing and Sustainable Societies (COMPASS) (COMPASS '21), June 28-fuly 02, 2021, Virtual Event, Australia. ACM, New York, NY, USA, 7 pages. https://doi.org/10.1145/3460112.3472312

\section{INTRODUCTION}

The etiology of diseases in later life may have a fetal origin and may be attributed to adverse effects of environmental exposures in utero because the embryonic period is the most critical period of development.[1] This causality theory is knowns as the Developmental Origins of Health and Disease or Baker hypothesis.[2] Previous systematic review studies have concluded that exposure to ambient outdoor air pollution, especially the Particulate Matter (PM) pollution, is one of the detrimental environmental factors that affect fetal development, and described associations between prenatal PM exposure and adverse birth outcomes including preterm birth ( $<37$ weeks of pregnancy) and low birth weight $(<2,500 \mathrm{~g})$. [35] The exact physiological mechanisms underlying PM affecting the fetal development have not been identified yet, but it has been suggested that its toxicity may be caused by the translocation of PM particles or components across the placenta, which activates inflammatory and oxidative responses and alters placental function.[6] In fact, one recent study proved that ambient black carbon particles can reach the fetal side of human placenta.[7]

Nepal and Bangladesh are low-income countries in South Asia that have undergone rapid urbanization in recent years. Due to the uncontrolled industrialization and old fleets of commercial vehicles characterized by inefficient combustion technologies, countries in 
South Asia have experienced the significant emission of air pollutants including PM2.5.[8] As a matter of fact, Nepal and Bangladesh ranked 145th and 162th respectively on air quality in the $2020 \mathrm{En}-$ vironmental Performance Index.[9] In Bangladesh, the geometric mean annual PM2.5 concentration increased from $33.8 \mu \mathrm{g} / \mathrm{m}^{3}$ in 1998 to $53.5 \mu \mathrm{g} / \mathrm{m}^{3}$ in 2014, exposing a population of about 160 million to ambient PM2.5 concentrations far exceeding the World Health Organization (WHO) guideline of $10 \mu \mathrm{g} / \mathrm{m}^{3}$.[10]

The prevalence of preterm birth and low birth weight is particularly high in low-income countries. It has been estimated that about 15 million preterm babies are born each year worldwide; $60 \%$ of them are born in South Asia and Sub-Saharan Africa.[11] Also, the prevalence of low birth weight is estimated to be $36 \%$ in Bangladesh[12] and 32\% in Nepal[13] while WHO reports that $15 \%$ to $20 \%$ of all births worldwide are low birth weight.[14]

Despite the high prevalence of preterm birth and low birth weight, and the growing evidence on associations of prenatal exposure to PM2.5 with adverse birth outcomes, no epidemiological studies have examined such associations in Nepal and Bangladesh mainly due to the lack of resources that affect PM2.5 exposure data collection. The Ministry of Environment of Bangladesh instituted air quality ground-monitoring stations in January 2014; however, they have been installed in only six locations, namely in Dhaka, Gazipur, Narayanganj, Chittagong, Sylhet, and Barisal, and therefore its coverage is still very limited.[15] Additionally, the government of Nepal started installing the air quality monitoring system in 2003; however, the number of reference-grade monitoring stations is only two in the country and is still limited.[16] As a result, using PM2.5 exposure data collected from these would restrict the populations and areas that we could study epidemiologically, increase uncertainty, and potentially introduce bias into health impact assessments. On the other hand, direct individual measurements using personal monitors are expensive, difficult to implement; hence, the number of study participants is limited and the length of time that exposures are measured. Due to these circumstances, a nationally representative epidemiological study that assesses the impact of PM2.5 exposure on the health of people has rarely been conducted in low-income countries including Nepal and Bangladesh.

This study aims at conducting a spatial-temporal analysis of an association between prenatal exposure to PM2.5 and preterm birth and term low birth weight in Nepal and Bangladesh by using the prediction model for PM2.5 daily concentrations that is geographically matched with Demographic and Health Survey (DHS) data. The objective of this study is three-fold: (1) Develop a prediction model for daily PM2.5 concentrations in Nepal and Bangladesh using ensemble machine learning and deep learning approaches incorporating satellite-detected air pollution data obtained from Sentinel-5 Precursor (Sentinel-5P), land use data, and meteorological data; (2) Estimate the PM2.5 levels for cluster locations included in the DHS Nepal 2021 and DHS Bangladesh 2021 by matching the developed prediction model with geocoordinates of DHS cluster locations; and (3) Examine the association between exposure to PM2.5 and preterm birth and term low birth weight in Nepal and Bangladesh using the DHS 2021 datasets. The main purpose of this poster is to introduce the overall picture of the study and present the materials, methods, and results of the first component of the study:
(1) Develop a prediction model for daily PM2.5 concentrations in Nepal and Bangladesh.

\section{DEVELOPMENT OF A PREDICTION MODEL}

\subsection{Background}

In recent years, a growing number of studies explored various statistical methods to predict the ground-level PM2.5 concentrations. In particular, satellite-detected Aerosol Optical Depth (AOD) observations, such as the Moderate Resolution Imaging Spectroradiometer (MODIS), has been widely used as a key predictor of ground-level PM2.5 concentrations.[17-20] AOD is a measure of the extinction of electromagnetic radiation at a given wavelength caused by the presence of aerosols in an atmospheric column.[21] On the contrary, satellite-based AOD is a measure of light attenuation in the column; therefore, it is largely affected by ambient conditions including vertical profile, presence of clouds, chemical composition, and humidity.[21] As a result, AOD have substantial missing data and these large numbers of data gaps limit the applicability of the product to estimating the ground-level PM2.5 concentrations.[22]

In light of the large number of missing data in AOD, many studies have proposed various methods in recent years. One strategy is to establish regional algorithms that are suited for local geographic and atmospheric conditions to retrieve more AOD pixels.[23] This strategy can significantly increase the coverage and potentially improve the accuracy of satellite-driven AOD; however, it is restricted to specific regions and cannot fill missing AOD data with true cloud coverage.[23] Another strategy is to use spatial statistical methods to estimate missing predicted concentrations of PM2.5 from the spatiotemporal autocorrelation. For example, one study used regional daily average PM2.5 levels and spatial smooth function to fill in missing PM2.5 predictions.[24] Although this method can improve the prediction coverage, but it cannot fill missing data when predicting historical PM2.5 concentrations before the establishment of air quality monitoring network because it relies on ground-based measurements from monitoring stations. Furthermore, it may demonstrate poorer performance if the monitoring networks are sparse.[23]

In this study, we propose a noble method that incorporates satellite-based air pollution data such as nitrogen dioxide $\left(\mathrm{NO}_{2}\right)$ and ozone $\left(\mathrm{O}_{3}\right)$ instead of AOD to spatially and temporally predict the ground-level PM2.5 concentrations in Nepal and Bangladesh where the air quality monitoring networks are sparse. Previous studies demonstrated that the presence of gaseous pollutants including $\mathrm{NO}_{2}$ and $\mathrm{O}_{3}$ is highly correlated with PM2.5 concentrations because the main sources of such pollutants are commonly represented by industrial activity, road traffic, and burnings of fossil fuels.[25] Our method also incorporated meteorological and land use information, and urbanization and human activity indicators to improve model performance because these are known to affect the dispersion and transport of PM2.5 and represent potential sources of PM2.5 and areas of higher concern. To author's knowledge, no previous studies developed a prediction model for daily PM2.5 concentrations using satellite-detected air pollution data instead of AOD. 


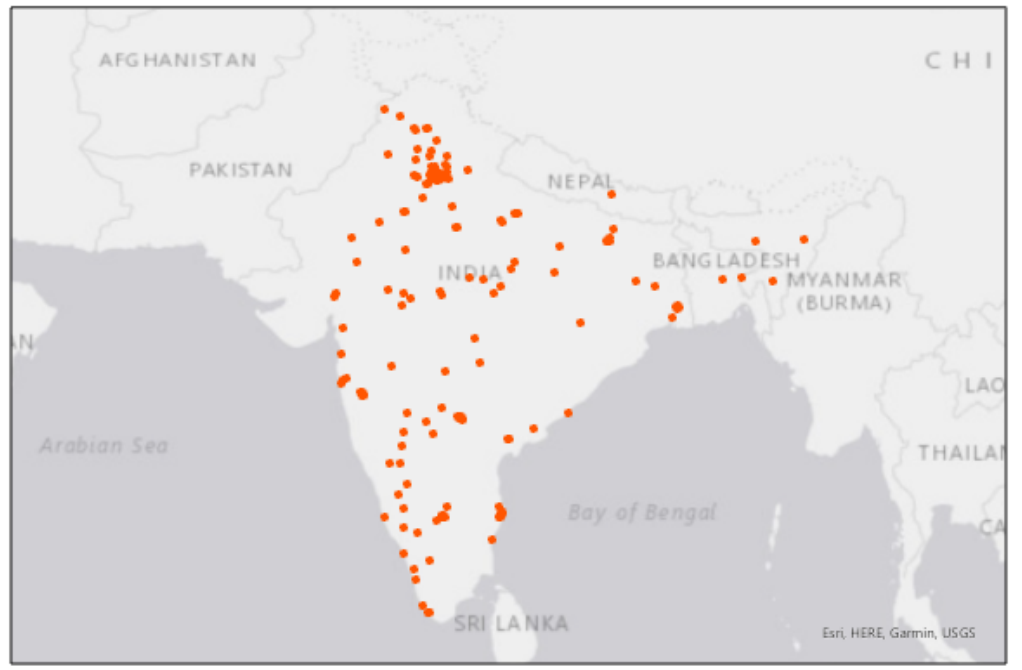

Figure 1: Location of monitoring stations.

\subsection{Materials and Methods}

This study developed a prediction model for daily PM2.5 concentrations in Bangladesh, and Nepal from November 22, 2018 to May 7, 2021. To optimize the predictions, we utilized three machine learning methods: XGBoost, LightGBM, and Deep Neural Network (DNN). The XGBoost and LightGBM are both a gradient boosting framework that uses tree-based learning algorithms. The DNN takes the input variable, processes them through various combinations and weights, and generates predictions.[26]

2.2.1 PM2.5 Data. Predicted daily PM2.5 was compared to groundmonitored PM2.5 concentrations to train the models and assess the accuracy of the various methods used. The ground monitoring PM2.5 data was obtained from the 'OpenAQ' website (https: //openaq.org), which aggregates ground monitoring data from public sources with a global coverage. Using the 'OpenAQ' API, we downloaded PM2.5 ground-monitored data from 214 referencegrade monitoring stations located in India, Bangladesh, and Nepal from November 22, 2018 to May 7, 2021 (Figure 1). The PM2.5 measures in India were included to train the models to increase the size of training data and therefore improve the model performance.

2.2.2 Input Variables and Data Sources. Using the geographic information of the reference-grade monitoring stations from which we obtained the ground-based PM2.5 concentrations, the air pollution data, meteorological information, land use data, and urbanization and human activity indicators were obtained from various sources using the Google Earth Engine API. Among these, daily mean values of air pollution and meteorological data were obtained for the same period as the ground-monitored PM2.5 data were acquired to account for temporal variability of the ground-measured PM2.5 data, and were used for training of the models. Additionally, we added the month, day of year, square of day of year, sine of day of year, and cosine of day of year in the models.
2.2.3 Air Pollution Data: The air pollution variables used in training the models were $\mathrm{NO}_{2}$ column number density $\left(\mathrm{mol} / \mathrm{m}^{2}\right)$, tropospheric $\mathrm{NO}_{2}$ column number density $\left(\mathrm{mol} / \mathrm{m}^{2}\right)$, stratospheric $\mathrm{NO}_{2}$ column number density $\left(\mathrm{mol} / \mathrm{m}^{2}\right), \mathrm{NO}_{2}$ slant column number density $\left(\mathrm{mol} / \mathrm{m}^{2}\right)$, absorbing aerosol index, carbon monoxide (CO) column number density $\left(\mathrm{mol} / \mathrm{m}^{2}\right)$, and $\mathrm{O}_{3}$ column number density $\left(\mathrm{mol} / \mathrm{m}^{2}\right)$. For all these pollutant data, the daily mean, maximum, and minimum values were collected. These air pollution variables were obtained from the Sentinel-5 Precursor (Sentinel-5P) space-borne satellite, which was launched on October 13, 2017. The Sentinel-5P is operated and managed by the European Commission under the "Copernicus" program and has spatial resolution of $7 \mathrm{x}$ $3.5 \mathrm{~km}^{2}$. The satellite operates in a sun-synchronous orbit at 824 kilometers and an orbital cycle of 16 days. The satellite carries a TROPOspheric Monitoring Instrument (TROPOMI) which provides a near-global coverage of air pollution caused by $\mathrm{NO}_{2}$ and other pollutants such as $\mathrm{O}_{3}, \mathrm{CO}$, and aerosols.[27]

2.2.4 Meteorological Data: The meteorological data included in training the models were temperature 2 meters above ground $\left({ }^{\circ} \mathrm{C}\right)$, specific humidity 2 meters above ground $(\mathrm{kg} / \mathrm{kg})$, relative humidity 2 meters above ground (\%), U component of wind speed $(\mathrm{m} / \mathrm{s}), \mathrm{V}$ component of wind speed $(\mathrm{m} / \mathrm{s})$, total cloud coverage $(\%)$, total precipitable water for entire atmosphere $\left(\mathrm{kg} / \mathrm{m}^{2}\right)$, pressure $(\mathrm{Pa})$. These variables were collected from the Global Forecast System, which is a weather forecast model produced by the National Centers for Environmental Prediction (NCEP). For temperature, specific humidity, relative humidity, and total cloud coverage, we collected the mean, maximum, and minimum daily values.

2.2.5 Land Use Data: We used Normalized Difference Vegetation Index (NDVI) and elevation (m) as land use information to train the models. NDVI was collected from the NOAA Climate Data Record. It contains gridded daily NDVI derived from the NOAA Surface Reflectance product. It provides a measurement of surface 
Table 1: Input variables selected by RFECV

\begin{tabular}{|c|c|c|}
\hline XGBoost & LightGBM & \\
\hline Light at night (mean) & Light at night (mean) & $\begin{array}{l}\text { Stratospheric } \mathrm{NO}_{2} \text { column number density } \\
\text { (mean) }\end{array}$ \\
\hline Population density (mean) & Population density (mean) & $\begin{array}{l}\text { Tropospheric } \mathrm{NO}_{2} \text { column number density } \\
\text { (mean) }\end{array}$ \\
\hline NDVI (mean) & NDVI (mean) & $\mathrm{O}_{3}$ column number density (mean, min) \\
\hline Elevation (mean) & Elevation (mean) & Day of year \\
\hline Human modification index (mean, min) & $\begin{array}{l}\text { Human modification index (mean, min, } \\
\max \text { ) }\end{array}$ & Day of year (sine) \\
\hline Specific humidity (mean) & Specific humidity (mean, min) & \\
\hline \multirow[t]{2}{*}{ Temperature (mean) } & Temperature (min, $\max )$ & \\
\hline & V component of wind (mean) & \\
\hline
\end{tabular}

vegetation coverage activity, gridded at a resolution of $0.05^{\circ}$ and computed globally over land surfaces.[28] The elevation data was obtained from the Global Multi-resolution Terrain Elevation Data 2010 .

2.2.6 Urbanization and Human Activity Indicators: The variables added as urbanization and human activity indicators were population density, light at night, and human modification index. We collected the mean, maximum, and minimum values for these variables. Population density was obtained from the Gridded Population of World Version 4 (GPWv4), which models the distribution of global human population for the years 2000, 2005, 2010, 2015, and 2020 on approximately $1 \mathrm{~km}$ grid cells. Mean annual light at night was obtained from the global radiance-calibrated nighttime lights version 4, defense meteorological program operational linescan system. Finally, the human modification index was collected from the global human modification dataset, which provides a cumulative measure of human modification of terrestrial lands globally at 1 $\mathrm{km}^{2}$ resolution. It incorporates 13 individual datasets on human settlement, agriculture, transportation, mining and energy production, and electrical infrastructure.[29]

2.2.7 Feature Selection. The presence of multicollinearity of input features was firstly assessed through creation of a correlation matrix. Subsequently, Variance Inflation Factor (VIF) was calculated to confirm the highly correlated variables. As a result, mean relative humidity and total precipitable water for entire atmosphere were excluded from the training models. The data were then split into training and test sets stratified by country. The training set had 50,832 data points and test set had 12,709 data points. Finally, the study took the Recursive Feature Elimination with Cross-Validation (RFECV) approach to select the features that can achieve the highest model performance. Table 1 summarizes the selected features as a result of RFECV for the XGBoost and LightGBM. The DNN returned the highest performance when all the input features were included in the model, except for the mean relative humidity and total precipitable water for entire atmosphere.

2.2.8 Hyper-Parameter Tuning. Machine learning methods require specification of hyper-parameters, parameters that control the learning process, to improve the model performance. This study used a
Table 2: Five-Fold Cross-Validation Results

\begin{tabular}{llll}
\hline Model & $\mathrm{R}^{2}$ & RMSE & MAE \\
\hline XGBoost & 0.758 & 24.89 & 16.19 \\
LightGBM & 0.775 & 24.38 & 15.62 \\
DNN & 0.770 & 24.79 & 15.13 \\
\hline
\end{tabular}

grid search and investigated the five-fold cross-validated $\mathrm{R}^{2}$, Root Mean Square Error (RMSE), and Mean Absolute Error (MAE) values to optimize the hyper-parameters for the XGBoost, LightGBM, and DNN. For the XGBoost, we tuned the learning rate, maximum depth, number of estimators, gamma, and L2 regularization term on weights. For the LightGBM, the following hyper-parameters were tuned: learning rate, maximum depth, number of leaves, number of estimators, boosting type, subsample, and L2 regularization term on weights. For the DNN, we used five hidden layers and tuned the number of neurons, the number of times the data is run through the network, the adaptative learning rate, and a shrinkage parameter.

\subsection{Results}

Table 2 summarizes the results of the five-fold cross-validation.

Taking the above cross-validated results into the consideration, the final model chosen was the LightGBM. Figure 2 shows the feature importance of input variables used in the LightGBM model. The most important features were day of year and elevation, followed by mean NDVI, mean stratospheric $\mathrm{NO}_{2}$ column number density, tropospheric $\mathrm{NO}_{2}$ column number density, and $\mathrm{O}_{3}$ column number density.

The final LightGBM model was used to generate daily PM2.5 predictions using the test set, and $\mathrm{R}^{2}$, RMSE, and MAE were calculated to confirm the model performance. As a result, $\mathrm{R}^{2}$ was 0.79 , RMSE was 23.78, and MAE was 15.34 (Figure 3).

This model performed differently during different seasons and worked reasonably well for a post-monsoon period (October to December) and winter (January to February). On the contrary, the model performed poorly for summer (March to May) and during a monsoon period (June to September). $\mathrm{R}^{2}$ was 0.79 for a postmonsoon period, 0.74 for winter, 0.61 for summer, and 0.46 for a 
[Poster] Association between prenatal exposure to ambient particulate matter (PM2.5) and adverse birth outcomes in Nepal and Bangladesh using the prediction model for daily PM2.5 concentrations matched with

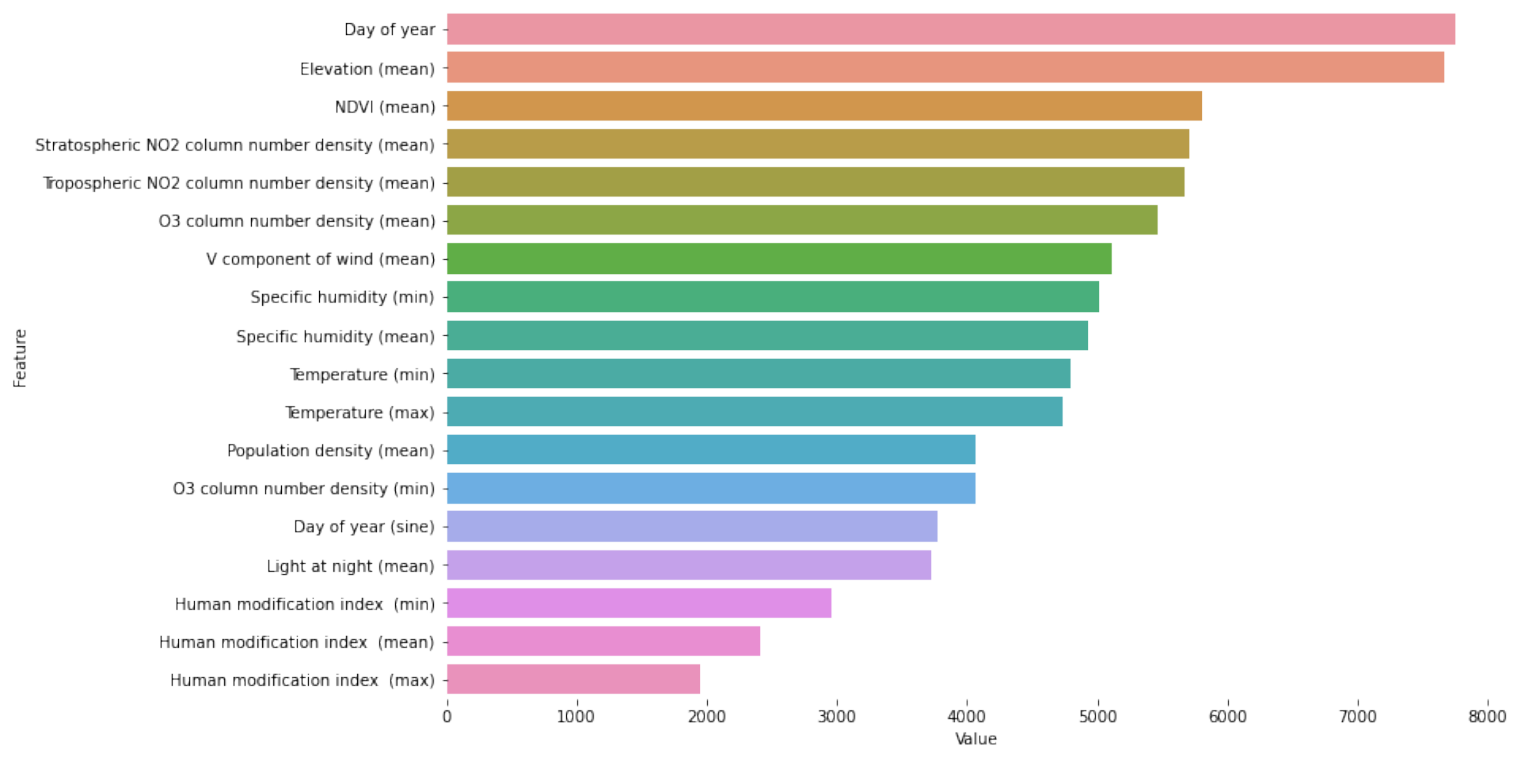

Figure 2: LightGBM variable importance.

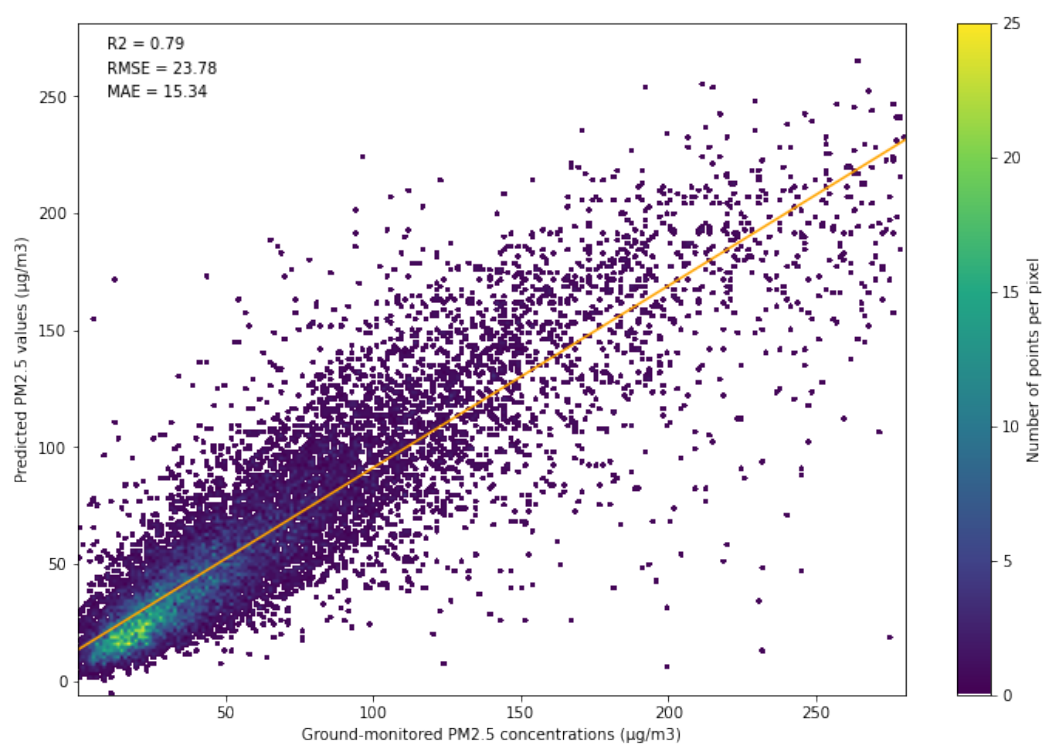

Figure 3: PM2.5 predictions generated by the final model using the test set.

monsoon period (Figure 4). The poor performance during a monsoon period may be associated with the lower quality of satellitedriven features that can be affected by local weather.

Finally, we confirmed that the model did not perform significantly differently for different countries. $\mathrm{R}^{2}$ was 0.79 for India and Bangladesh, and 0.72 for Nepal. Figure 5 demonstrates the model performance for Bangladesh and Nepal as well as the changes in PM2.5 predictions over time for each country.

\section{DISCUSSION AND CONCLUSION}

In this study, we developed a daily PM2.5 prediction model that can be used for regions that have sparse air quality monitoring networks by incorporating the satellite-detected air pollution data, meteorological data, land use information, and urbanization indicators into the model. Our model has shown to be a reasonably accurate and robust measure of PM2.5 levels in South Asia, suggesting that Sentinel-5P data have considerable potential to provide predictive power for predictions of PM2.5 concentrations. 

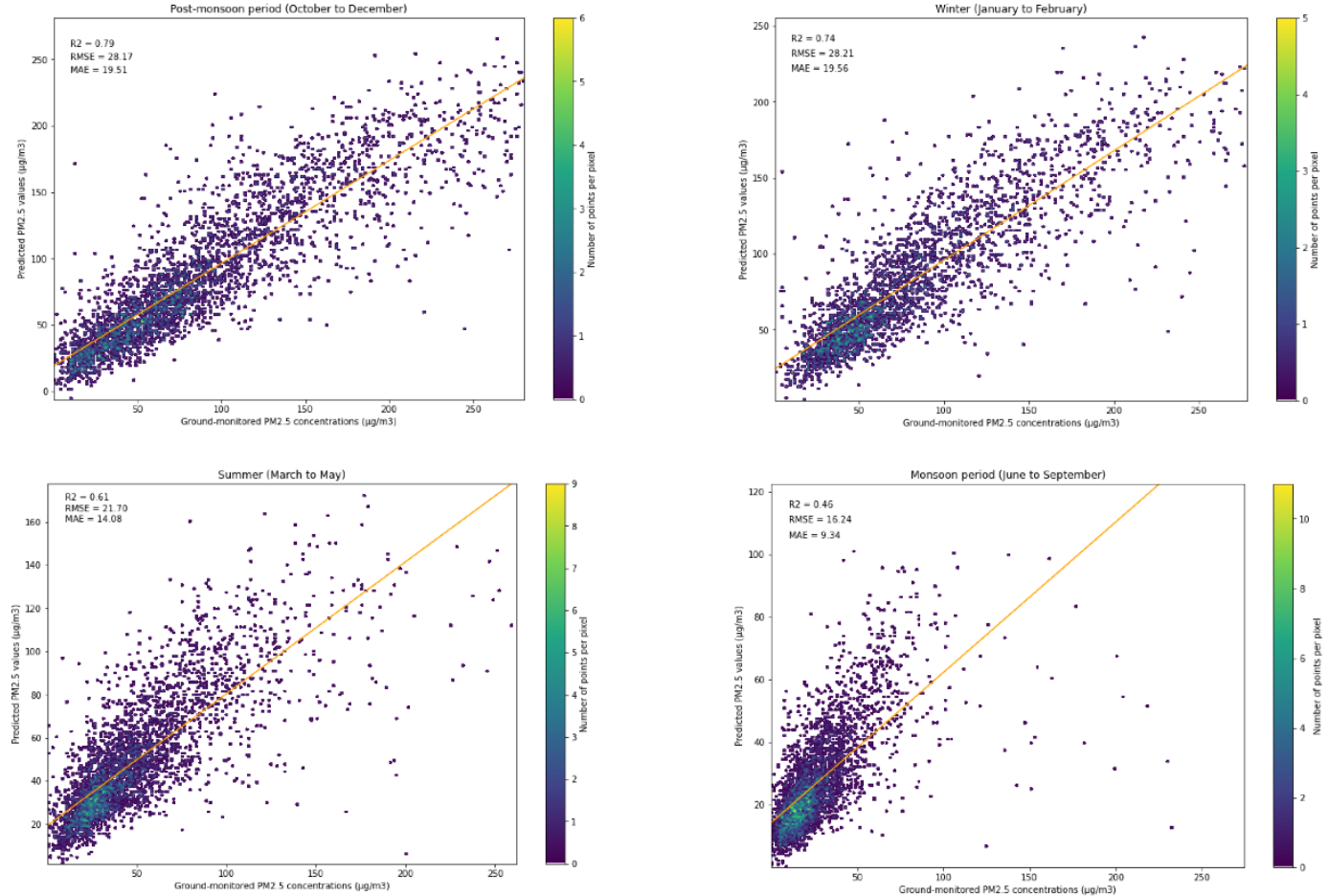

Figure 4: Model performance during different seasons.
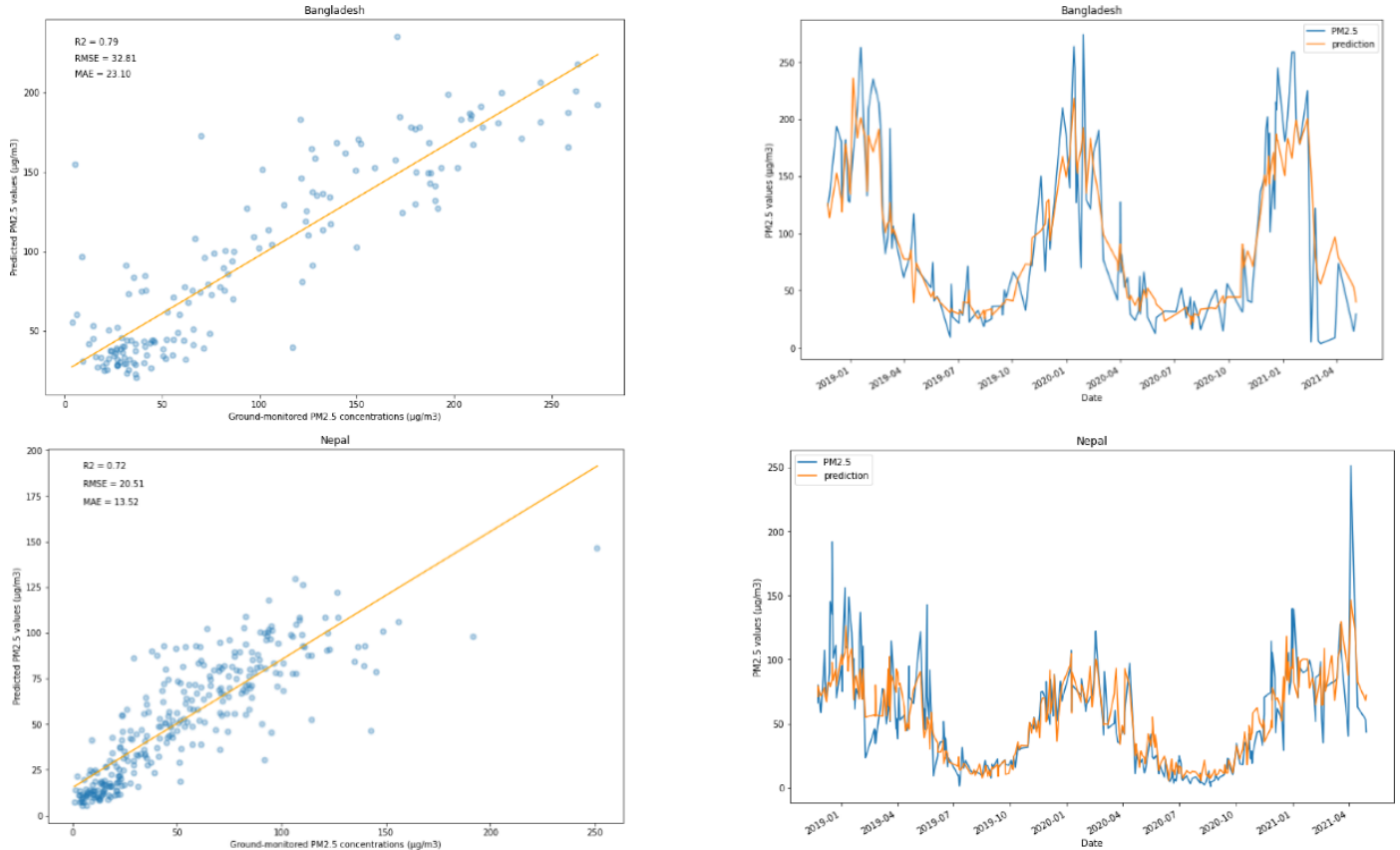

Figure 5: Model performance and temporal prediction changes for Bangladesh and Nepal. 
[Poster] Association between prenatal exposure to ambient particulate matter (PM2.5) and adverse birth outcomes in Nepal and Bangladesh using the prediction model for daily PM2.5 concentrations matched with demographic and health survey data: spatio-temporal analysis

However, our model still needs to be improved especially considering the weak $\mathrm{R}^{2}$ values for summer and monsoon period. One strategy is to add input variables that are highly correlated with PM2.5 concentrations in the countries. For instance, our model was not able to incorporate the distance to sources of air pollution such as distance to nearest major road, airport, and power plant. The traffic-related data such as traffic counts are also considered to be an important indicator of PM2.5 concentrations. Additionally, adding the ground-level $\mathrm{NO}_{2}$ and $\mathrm{O}_{3}$ to input variables could greatly contribute to improving the model performance. For countries that have limited monitoring networks such as Nepal and Bangladesh, such ground-level concentrations may be statistically computed from $\mathrm{NO}_{2}$ and $\mathrm{O}_{3}$ column values obtained from Sentinel-5P.

Using the developed prediction model, we can achieve the overall research goal - to investigate the association between prenatal exposure to PM2.5 and adverse birth outcomes such as preterm birth and low birth weight in Nepal and Bangladesh. When the DHS Nepal 2021 and DHS Bangladesh 2021 datasets become available, PM2. 5 concentrations can be estimated for cluster locations included in the DHS datasets, and the predicted values can be used for an epidemiological study that investigates the effect of PM2.5 on adverse birth outcomes in Bangladesh and Nepal.

\section{ACKNOWLEDGMENTS}

This study received no specific grant from any funding agency in the public, commercial, or not-for-profit sectors.

\section{REFERENCES}

[1] Kenner C. Fetal Development: Environmental Influences and Critical Periods [Internet]. Springer Publishing Company; 2021 [cited 2021 May 26]. Available from: https://connect.springerpub.com/content/book/978-0-8261-3914-6/part/ part01/chapter/ch01.

[2] Barker DJP. Fetal origins of coronary heart disease. BMJ. 1995;311:171-174.

[3] Lamichhane DK, Leem J-H, Lee J-Y, et al. A meta-analysis of exposure to particulate matter and adverse birth outcomes. Environ Health Toxicol [Internet] 2015 [cited 2021 Feb 24];30. Available from: https://www.ncbi.nlm.nih.gov/pmc/ articles/PMC4722965/.

[4] Sapkota A, Chelikowsky AP, Nachman KE, et al. Exposure to particulate matter and adverse birth outcomes: a comprehensive review and meta-analysis. Air Qual Atmosphere Health. 2012;5:369-381.

[5] Stieb DM, Chen L, Eshoul M, et al. Ambient air pollution, birth weight and preterm birth: A systematic review and meta-analysis. Environ Res. 2012;117:100-111.

[6] Steinle S, Johnston HJ, Loh M, et al. In Utero Exposure to Particulate Air Pollution during Pregnancy: Impact on Birth Weight and Health through the Life Course. Int J Environ Res Public Health [Internet]. 2020 [cited 2021 May 26];17. Available from: https://www.ncbi.nlm.nih.gov/pmc/articles/PMC7730886/.

[7] Bové H, Bongaerts E, Slenders E, et al. Ambient black carbon particles reach the fetal side of human placenta. Nat Commun. 2019;10:3866.

[8] Shakya KM, Rupakheti M, Aryal K, et al. Respiratory Effects of High Levels of Particulate Exposure in a Cohort of Traffic Police in Kathmandu, Nepal. J Occup Environ Med. 2016;58:e218.

[9] Zachary A W, John W. E, Alex de S, et al. 2020 Environmental Performance Index. [Internet]. New Haven, CT: Yale Center for Environmental Law \& Policy; 2020 [cited 2021 May 26]. Available from: https://epi.yale.edu/downloads/
COMPASS '21, June 28-July 02, 2021, Virtual Event, Australia

epi2020report20210112.pdf.

[10] Goyal N, Canning D. Exposure to Ambient Fine Particulate Air Pollution in Utero as a Risk Factor for Child Stunting in Bangladesh. Int J Environ Res Public Health. 2018;15:22.

[11] Baqui AH, Rosen HE, Lee ACC, et al. Preterm birth and neonatal mortality in a rural Bangladeshi cohort: implications for health programs. J Perinatol. 2013;33:977-981.

[12] Grantham-McGregor SM. Effects of prenatal food and micronutrient supplementation on infant development: a randomized trial from the Maternal and Infant Nutrition Interventions, Matlab (MINIMat) study. [cited 2021 May 26]; Available from: https://core.ac.uk/reader/79608952?utm source=linkout.

[13] Sharma SR, Giri S, Timalsina U, et al. Low Birth Weight at Term and Its Determinants in a Tertiary Hospital of Nepal: A Case-Control Study. PLOS ONE. 2015;10:e0123962.

[14] World Health Organization. Global Nutrition Targets 2025, Low Birth Weight Policy Brief [Internet]. 2014 [cited 2021 May 26]. Available from: http://apps.who. int/iris/bitstream/handle/10665/149020/WHO_NMH_NHD_14.5_eng.pdf.

[15] Mahmood A, Hu Y, Nasreen S, et al. Airborne Particulate Pollution Measured in Bangladesh from 2014 to 2017. Aerosol Air Qual Res. 2019;19:272-281.

[16] Edwards L, Rutter G, Iverson L, et al. Personal exposure monitoring of PM2.5 among US diplomats in Kathmandu during the COVID-19 lockdown, March to June 2020. Sci Total Environ. 2021;772:144836.

[17] Donkelaar A van, Martin RV, Park RJ. Estimating ground-level PM2.5 using aerosol optical depth determined from satellite remote sensing. J Geophys Res Atmospheres [Internet]. 2006 [cited 2021 May 27];111. Available from: https: //agupubs.onlinelibrary.wiley.com/doi/abs/10.1029/2005JD006996.

[18] Gupta P, Christopher SA, Wang J, et al. Satellite remote sensing of particulate matter and air quality assessment over global cities. Atmos Environ. 2006;40:58805892.

[19] Hoff RM, Christopher SA. Remote Sensing of Particulate Pollution from Space: Have We Reached the Promised Land? J Air Waste Manag Assoc. 2009;59:645-675.

[20] Chang HH, Hu X, Liu Y. Calibrating MODIS aerosol optical depth for predicting daily PM2.5 concentrations via statistical downscaling. J Expo Sci Environ Epidemiol. 2014;24:398-404.

[21] Kloog I, Chudnovsky AA, Just AC, et al. A New Hybrid Spatio-Temporal Model For Estimating Daily Multi-Year PM2.5 Concentrations Across Northeastern USA Using High Resolution Aerosol Optical Depth Data. Atmospheric Environ Oxf Engl 1994. 2014;95:581-590.

[22] Yang J, Hu M. Filling the missing data gaps of daily MODIS AOD using spatiotemporal interpolation. Sci Total Environ. 2018;633:677-683.

[23] Xiao Q, Wang Y, Chang HH, et al. Full-coverage high-resolution daily PM2.5 estimation using MAIAC AOD in the Yangtze River Delta of China. Remote Sens Environ. 2017;199:437-446.

[24] Just AC, Wright RO, Schwartz J, et al. Using High-Resolution Satellite Aerosol Optical Depth To Estimate Daily PM2.5 Geographical Distribution in Mexico City. Environ Sci Technol. 2015;49:8576-8584.

[25] Beckerman B, Jerrett M, Brook JR, et al. Correlation of nitrogen dioxide with other traffic pollutants near a major expressway. Atmos Environ. 2008;42:275-290.

[26] Glorot X, Bordes A, Bengio Y. Deep Sparse Rectifier Neural Networks. Proc Fourteenth Int Conf Artif Intell Stat [Internet]. JMLR Workshop and Conference Proceedings; 2011 [cited 2021 May 27]. p. 315-323. Available from: http://proceedings.mlr.press/v15/glorot11a.html.

[27] Veefkind JP, Aben I, McMullan K, et al. TROPOMI on the ESA Sentinel-5 Precursor: A GMES mission for global observations of the atmospheric composition for climate, air quality and ozone layer applications. Remote Sens Environ. 2012;120:70-83.

[28] Eric V, Chris J, Ivan C, et al. NOAA Climate Data Record (CDR) of Normalized Difference Vegetation Index (NDVI), Version 4. NOAA National Climatic Data Center. [Internet]. 2014 [cited 2021 May 27]. Available from: https://www.ncei. noaa.gov/access/metadata/landing-page/bin/iso?id=gov.noaa.ncdc:C00813.

[29] Kennedy CM, Oakleaf JR, Theobald DM, et al. Managing the middle: A shift in conservation priorities based on the global human modification gradient. Glob Change Biol. 2019;25:811-826. 